\title{
Few Insights on the problem of COVID-19
}

\author{
El Hassoun $\mathrm{O}^{1}$, Valaskova $\mathrm{Z}^{2}$, Polak $\mathrm{S}^{1}$, Hulin $\mathrm{I}^{3}$ \\ Institute of Histology and Embryology, Faculty of Medicine, Comenius University, \\ Bratislava, Slovakia. olia.elhassoun@fmed.uniba.sk
}

\section{ABSTRACT}

The recent Coronavirus 2019 outbreak took the world by surprise and called for global drastic measures. At this early point in the timeline of the pandemic, several questions remain open until the results of large scale studies become available. This article offers few insights on scattered issues; including the clinical characteristics, pathology and diagnosis, as well as treatment perspectives and public health approach. Focusing healthcare resources on necessary treatment and prevention and combining efforts for developing feasible solutions will be decisive for time needed to achieve worldwide containment (Tab. 1, Ref. 23). Text in PDF www.elis.sk

KEY WORDS: COVID-19, Coronavirus 2019, pandemic, public health.

\section{Introduction}

Pneumonia associated with a novel coronavirus emerged in Wuhan in China (1). This novel coronavirus (CoV19), also known as COVID-19, SARS-CoV2 or Wuhan pneumonia, was first reported in December 2019. Since then it has rapidly progressed to become a major global public health issue (21).

\section{The CoV19 virus}

Coronaviruses are enveloped positive strand RNA viruses, they belong to the subfamily of Orthocoronavirinae (13). They are divided into four sub groups; alfa, beta gamma and delta (9). The coronavirus family essentially causes zoonotic infections in birds and mammals (13), with documented outbreaks of disease in cattle and other animals $(4,15)$. It is also known to cause mainly respiratory tract infections in humans (13). CoV19 is the seventh coronavirus known to infect humans. The first four; 229E, NL63, OC43 and HKU1 cause mild upper respiratory tract infections. The remaining two are the SARS-CoV which causes severe acute respiratory syndrome, and the MERS-CoV which can cause atypical pneumonia (6). CoV19 similar to SARS-CoV belogns to the group of betacoronaviruses (7). By February 2020, CoV19 has caused more fatalities than SARS-CoV and MERS-CoV combined (11). A decisive moment in the dynamics of CoV19 propagation is acquiring the ability of human to human spread.

${ }^{1}$ Institute of Histology and Embryology, Faculty of Medicine, Comenius University, Bratislava, Slovakia, ${ }^{2}$ Institute of Pathophysiology, Faculty of Medicine, Comenius University, Bratislava, Slovakia, and ${ }^{3}$ Faculty of Medicine, Comenius University, Bratislava, Slovakia

Address for correspondence: El Hassoun Olia, Institute of Histology and Embryology, Faculty of Medicine, Comenius University, Bratislava, Slovakia
The origin of the CoV19 is believed to be a local wet market, where wild animals were captured and sold (19). Phylogenetic analysis of CoV19 suggests a bat origin with a diversity of possible intermediate hosts (10). The former SARS-CoV was also demonstrated to have a bat reservoir. The syndrome named Middle East Respiratory Syndrome (MERS) Coronavirus infection is documented in camels since 1992. The first documented introduction to humans was in 2011. Camels were proposed as potential reservoirs for human transmission, however, possible spread of infection from humans to camels or via another host to both species was not concluded. (4)

\section{Clinical characteristics and diagnosis}

Clinical manifestations of the infection are summarised in Table 1, alongside other characteristics of small cohorts published from various Chinese hospitals. Interestingly enough, and even though by the time of writing this article, fatalities in Europe have surpassed those in China, no studies on European patients have yet been found in available literature. For this reason, a lenient approach to the clinical manifestations bearing in mind regional and cohort differences must be warranted. This is especially true when dealing with the significantly older population of Europe in comparison to China. The relatively high mortality rate reported in the initial studies is partially due to them including only severe cases requiring hospitalisation. Statistics including asymptomatic and mild cases which do not require medical attention show a much lower mortality rate (6). Predicting the risk of mortality appeared to be in keeping with the MuLBSTA score (1). Children are seldom infected with CoV19, as well as SARS-CoV. It is suggested that RNA vaccines and their adjuvants enhance children immunity against this virus (13).

Several published guidelines for the initial diagnosis of CoV19 infection require the presence of fever and respiratory symptoms 
Tab. 1. A summary of clinical and baseline characteristics of few descriptive studies.

\begin{tabular}{lccc}
\hline Clinical signs & Chen et al (1) & Wang et al (5) & Yang et al (19) \\
\hline Fever & $83 \%$ & $98.6 \%$ & $76.5 \%$ \\
Cough & $82 \%$ & $59.4 \%$ & $58.4 \%$ \\
Fatigue & - & $69.6 \%$ & - \\
Dyspnoea/ tight chest & $31 \%$ & $31.2 \%$ & 1.3 and $10.7 \%$ resp. \\
Muscle pain & $11 \%$ & $34.8 \%$ & $3.4 \%$ \\
Headache & $8 \%$ & $6.5 \%$ & $8.7 \%$ \\
Sore throat & $5 \%$ & $17.4 \%$ & $14.1 \%$ \\
Chest pain & $2 \%$ & - & $3.4 \%$ \\
Diarrhoea & $2 \%$ & $10.1 \%$ & $7.4 \%$ \\
Nausea and vomitting & $1 \%$ & 10.1 and $3.6 \%$ resp. & $1.3 \%$ \\
\hline Study characteristics & & & \\
\hline Number of patients & 99 & 138 & 149 \\
Age range & $21-82$ & $22-92$ & Average 45.11 \\
Duration of follow up & Up to 25 days & 1 month & Up to 1 month \\
Percentage of Men & $68 \%$ & $54.3 \%$ & $54.4 \%$ \\
Developed ARDS & $17 \%$ & 19.6 & $0 \% *$ \\
Mortality rate & $11 \%$ & $4.3 \%$ & $0 \%$ \\
\hline
\end{tabular}

* ARDS as a complication was not directly stated in the article, indirect indicator is $0 \%$ patients requiring intensive care.

in addition to positive epidemiological history (6). However, a high index to suspicion and caution is warranted, especially as positive travel history to flag potential CoV19 infection might not be very obvious after few generations of spread in a new country (8). Some reported cases demonstrated patients with no fever throughout the disease course (17) or patients with retrograde diagnosis indicating prolonged period before the manifestation of clinical symptoms (17).

The most reliable method for confirming the diagnosis of CoV19 infection is real time PCR. Test specimens are most commonly collected from the upper airways as nasopharyngeal or oropharyngeal swabs. These specimens must be kept in viral transport medium. Less commonly, sputum and bronchoalveolar lavage can be collected. CDC is recently recommending to collect only nasopharyngeal swabs, this is done by inserting a swab into the nostril parallel to the palate and leaving it for few seconds to absorb secretions (3). The possibility of negative swab PCR in the presence of pneumonia could be due to absence of the virus in the upper respiratory tract (17).

\section{Pathology and disease mechanisms}

CoV19 and SARS-CoV share similar coding regions for the spike protein (10) which plays a key role in mediating virus fusion and entering to host cell. Consequently, CoV19 and SARS-CoV utilize the same main host receptor, which is the angiotensin converting enzyme 2 (ACE2) (7). The CoV19 spike surface protein has a strong binding affinity to human ACE2 receptor protein. This receptor is abundant in type 2 alveolar epithelial cells, making them a reservior for viral replication (10). Studies on SARS-CoV which could probably be applicable to CoV19 shown that virus binding on ACE2 receptors caused its down-regulation, which in turn leads to the excessive production of angiotensin and the latter contributes to lung injury by increasing vascular permeability (11).

The virus appears to spread mainly through aerosol/droplet mechanism $(6,21)$. The ACE2 receptor however is expressed in several tissues, including high expression in the luminal intestinal surface. Based on the latter, the intestine was predicted to be also a possible major entry site for the virus (10). This prediction is not supported by the general respiratory imprint of the epidemic. Studies in Table 1 still showed a minor percentage of patients presenting with gastrointestinal symptoms, although it is not clear whether these are mutually exclusive with respiratory symptoms. Variability in tissue tropism of receptor mediated infection could be related to polymorphism of receptor expression in the population.

Peripheral leukopenia is noted in patients who later develop ARDS (2) The absolute value of lymphocytes in most patients was reduced (1). This was suggested to be a possible clue for early diagnosis (17). A comparison of the dynamics of laboratory parameters between infection survivors and non survivors showed lower lymphocyte count in non survivors (5), these results remain limited due to small sample size.

The overall health burden of a CoV19 infection must take into consideration extrapulmonary manifestations, and acute and chronic cardiac complications (14). In general, most common complications of viral pneumonia include bacterial concomitant infection, acute respiratory failure, cardiovascular collapse, sepsis and multiorgan failure and acute respiratory distress syndrome (20). Aside from the obvious known risk factors, patient stratification and risk prediction remain a problem.

Pathology examination of early phase CoV19 pneumonia in asymptomatic patients who were treated with lobectomy for adenocarcinoma revealed signs of acute interstitial pneumonia. These included lung edema, interstitial fibroblastic hyperplasia, type II pneumocyte hyperplasia and multinucleated giant cells in alveolar spaces. The patient who demonstrated focal fibrin clusters mixed with mononclear cells and suspected viral inclusions within type II pneumocytes, continued to deteriorate and eventually passed away while the other patient recovered and was discharged (17). No conclusive clues can be deduced from this case report besides a more advance stage of disease in the deceased patient at the time of sampling.

Post mortem examination of a CoV19 case demonstrated a picture of fulminant diffuse alveolar damage in lung samples in keeping with acute respiratory distress syndrome on the basis of viral pneumonia. Findings included formation of hyaline pseudomembranes and desquamation of pneumocytes. The inflammatory infiltrate was predominantly lymphocytic, with formation of multinucleated syncytial cells and atypical pneumocytes with viral cytopathic-like changes in the alveolar spaces (16).

\section{Treatment perspectives}

No fully proven and specific treatment for Cov19 exists (2). Supportive treatment includes various forms of ventilation aid (22). 
General supportive treatment includes nutritional interventions such as vitamins and minerals, in addition to immunoenhancers such as interferons and intravenous gammaglobulin (13). Antivirals like emdesivir and lopinavir/ritonavir are being evaluated against CoV19 after showing promising results in animal models of SARS-CoV and MERS-CoV (6). Several publications dedicated to CoV19 infection control and critical care recommendations are available (8).

There are several approaches to address the ACE2 mediated CoV19 infection, including blockage of the ACE2 receptor and delivering excessive soluble form of ACE2. A recombinant human ACE2 (rhACE2) has been found safe in healthy volunteers and a small cohort of patients with ARDS. A pilot trial rhACE2 on patients with severe CoV19 was initiated (10). The use of available angiotensin receptor 1 (AT1R) blockers such as losartan was suggested for reducing the aggressiveness and mortality of CoV19 (11). Potent targeted fusion/entry inhibitors against the SARS-CoV showed promising results against CoV19 (7).

In China, the use of the antimalarial medication chloroquine phosphate has shown potent activity against CoV19. Chloroquine phosphate has known anti-inflammatory effect. Its broad spectrum antiviral activity is believed to be based on increasing endosomal $\mathrm{pH}$ required for virus/cell fusion and interfering with glycolysation of cellular receptors of SARS-CoV (12). Further suggested coronavirus-specific treatment includes coronaviral protease inhibitors such as chymotrepsin 3 like (C3 like) inhibitors and papain like protease (PLP) inhibitors. These interfere with viral replication and also show inhibition of host innate immune functions. Both inhibitors showed promising results against SARS-CoV and are more than likely to be effective against CoV19 (13). All these potential treatments still await validation studies.

Future investigation of the course of CoV19 infection in relation to the drug profile of patients with comorbidities might reveal some insights on the behaviour of the virus and help further stratify risk groups. This can be especially interesting in cardiac patients who are already on ACE inhibitors or angiotensin receptor inhibitors. Future studies will also clarify the peculiarities of European and Asian CoV19 strains, which could provide the substrate to further explain the dynamics of spread in a given region.

\section{Public health issues}

As unfortunate as the pandemic might appear, simple preventive measures are the most effective. Many governments have taken appropriate steps to contain disease spread, with clear instructions and detailed information available for the public. There is no doubt that this pandemic has developed into a global healthcare crisis, which demands regulation of healthcare providers through legislatives. Never in history have such regulations been implemented at this large scale. A stochastic transmission model was developed to quantify the potential effectiveness of contact tracing and isolation of cases for disease control. It showed that in most scenarios, effective measures are enough to control a new outbreak within 3 months (23).
It is the duty of scientists and healthcare professionals to engage to the best of their knowledge with legislators for the formation and implementation of population-tailored public health plan.

Surveillance by full population testing seems hardly like a cost-effective approach, especially when population lock-down measures are already in place. On the contrary, massive testing can cause panic in cases that otherwise do not require medical attention. An alternative surveillance approach suggested for pandemics when cases are too numerous can be adopted; where algorithms are designed to ascertain a number of subjects for testing with highly sensitive but non specific symptoms. The yielding percentage of positive cases will provide an estimate of case burden (18).

Targeted testing to confirm CoV19 origin of pneumonia is warranted once specific targeted treatment is available. Till then, and in the context of insufficient testing capacity, diagnosis based on symptoms and exposure will have to suffice. Only time will show whether drastic measures are substantiated in low risk populations, such as school children. Meanwhile, focusing healthcare in the time of crisis on those who need it, in addition to responsible conduct in the form of isolation, quarantine and social distancing continue to be the best possible option for disease containment.

\section{References}

1. Chen N, Zhou M, Dong X, Qu J, Gong F, Han Y, ... Yu T. Epidemiological and clinical characteristics of 99 cases of 2019 novel coronavirus pneumonia in Wuhan, China: a descriptive study. The Lancet 2020; 395 (10223): 507-513.

2. Wang F S, Zhang C. What to do next to control the 2019-nCoV epidemic? The Lancet 2020; 395 (10222): 391-393.

3. Clinical Specimens: Novel Coronavirus (2019-NCoV). Centers for Disease Control and Prevention, Centers for Disease Control and Prevention, 14 Mar. 2020, www.cdc.gov/coronavirus/2019-ncov/lab/guidelinesclinical-specimens.html.

4. Alagaili AN, Briese T, Mishra N, Kapoor V, Sameroff SC, de Wit E, ... Epstein J H. Middle East respiratory syndrome coronavirus infection in dromedary camels in Saudi Arabia. MBio 2014; 5 (2): e00884-14.

5. Wang D, Hu B, Hu C, Zhu F, Liu X, Zhang J, ... Zhao Y. Clinical characteristics of 138 hospitalized patients with 2019 novel coronavirusinfected pneumonia in Wuhan, China. Jama 2020.

6. Bassetti M, Vena A, Giacobbe DR. The Novel Chinese Coronavirus (2019-nCoV) Infections: challenges for fighting the storm. Eur J Clin Invest 2020; 50 (3): e13209.

7. Xia S, Zhu Y, Liu M, Lan Q, Xu W, Wu Y, ... Lu L. Fusion mechanism of 2019-nCoV and fusion inhibitors targeting HR1 domain in spike protein. Cell Mol Immunol 2020; 1-3.

8. Wax RS, Christian MD. Practical recommendations for critical care and anesthesiology teams caring for novel coronavirus (2019-nCoV) patients. Canad J Anesth J Canad d'anesthésie 2020; 1-9.

9. Kumar D, Malviya R, Sharma PK. Corona Virus: A Review of COVID-19.

10. Zhang H, Penninger JM, Li Y, Zhong N, Slutsky AS. Angiotensinconverting enzyme 2 (ACE2) as a SARS-CoV-2 receptor: molecular mechanisms and potential therapeutic target. Intens Care Med 2020; $1-5$. 
471-474

11. Gurwitz D. Angiotensin receptor blockers as tentative SARS-CoV-2 therapeutics. Drug Develop Res 2020.

12. Gao J, Tian Z, Yang X. Breakthrough: Chloroquine phosphate has shown apparent efficacy in treatment of COVID-19 associated pneumonia in clinical studies. Biosci Trends 2020.

13. Zhang L, Liu Y. Potential interventions for novel coronavirus in China: a systematic review. J Med Virol 2020.

14. Xiong TY, Redwood S, Prendergast B, Chen M. Coronaviruses and the cardiovascular system: acute and long-term implications. Euro Heart J 2020.

15. Yurov KP, Alekseenkova SV, Pchelnikov AV, Mnikova LA, Ishkova TA, Yurov GK. Modern approach to diagnostics of respiratory cattle infections caused by Corona and Herpesviruses. Veter Med 2013; (8): 23-29.

16. Xu Z, Shi L, Wang Y, Zhang J, Huang L, Zhang C, ... Tai Y. Pathological findings of COVID-19 associated with acute respiratory distress syndrome. The Lancet Resp Med 2020.

17. Tian S, Hu W, Niu L, Liu H, Xu H, Xiao SY. Pulmonary Pathology of Early Phase SARS-COV-2 Pneumonia 2020.
18. Lipsitch M, Swerdlow DL, Finelli L. Defining the epidemiology of Covid-19 - studies needed. New Engl J Med 2020.

19. Yang W, Cao Q, Qin L, Wang X, Cheng Z, Pan A, ... Yan F. Clinical characteristics and imaging manifestations of the 2019 novel coronavirus disease (COVID-19): A multi-center study in Wenzhou city, Zhejiang, China. J Infection 2020.

20. Freeman AM, Soman-Faulkner K, Leigh Jr TR. Viral Pneumonia. In: StatPearls [Internet]. StatPearls Publishing 2019.

21. Lai CC, Shih TP, Ko WC, Tang HJ, Hsueh PR. Severe acute respiratory syndrome coronavirus 2 (SARS-CoV-2) and corona virus disease-2019 (COVID-19): the epidemic and the challenges. Internat J Antimicrob Agents 2020; 105924.

22. Xie J, Tong Z, Guan X, Du B, Qiu H, Slutsky AS. Critical care crisis and some recommendations during the COVID-19 epidemic in China. Intens Care Med 2020; $1-4$.

23. Hellewell J, Abbott S, Gimma A, Bosse NI, Jarvis CI, Russell TW, ... Flasche S. Feasibility of controlling COVID-19 outbreaks by isolation of cases and contacts. The Lancet Global Health 2020.

Received Marec 30, 2020.

Accepted April 20, 2020. 